\title{
Medication-Related Osteonecrosis of the Jaws (MRONJ). Case Report And Update.
}

\author{
Jorge A. Cano-Durán ${ }^{1}$, Daniel Ortega-Concepción ${ }^{1}$, Juan Francisco Peña- \\ Cardelles ${ }^{1}$, José González-Serrano ${ }^{2}$, Mariano García-Riart ${ }^{2}$, Víctor M. Paredes- \\ Rodríguez ${ }^{3}$, Juan López-Quiles ${ }^{4}$. \\ ${ }^{1}$ DDS. Oral Medicine Postgraduate. School of Dentistry of the Complutense University of Madrid. \\ ${ }^{2}$ DDS. Oral Medicine Postgraduate. Postgraduate student in Master degree program of Oral Surgery and \\ Implantology. School of Dentistry of the Complutense University of Madrid. \\ ${ }^{3}$ DDS, phD. Master degree program in Oral Surgery and Implantology. Honorary professor in the Department \\ of Medicine and Orofacial surgery. School of Dentistry of the Complutense University of Madrid. \\ ${ }^{4}$ DDS. MD. phD. Specialist in Oral and Maxillofacial Surgery. Professor contracted doctor of the Department \\ of Medicine and Orofacial surgery. School of Dentistry of the Complutense University of Madrid.
}

\begin{abstract}
:
Background. Antiresorptive drugs are used to prevent bone resorption derived from different diseases. One of the most frequent complications of these drugs is the osteonecrosis of the jaws (ONJ).

Case report. We report the case of an 82-year-old male presenting multiple ONJ lesions in the maxilla associated with the trauma of a removable partial denture and the bisphosphonates intake.

Discussion. The main controversy of ONJ lesions is the treatment that should be performed. Although it has been seen that there is no a totally effective therapy for ONJ, currently there are some promising and noninvasive techniques that seem to be effective, improving the quality of life of the patient.
\end{abstract}

Keywords: Osteonecrosis, Jaws, Bisphosphonates, Denosumab, Treatment.

\section{Introduction}

Antiresorptive drugs modulate bone metabolism and prevent bone resorption. They are used in Paget's disease, osteoporosis or for the control of bone metastases as occurs in prostate or breast cancers. Among these drugs we find Bisphosphonates (BFs) and monoclonal antibodies such as Denosumab [1].

Despite the efficacy of these drugs in the control of these pathologies, these are not free of complications, being the Osteonecrosis of the Jaws (ONJ) one of the most studied due to their severity. Initially ONJ was only related to the use of intravenous BFs. However, over the years and the greater knowledge of the disease and associated pharmacology, it has been verified not only its correlation with the use of oral BFs, but also with the use of other antiresorptive drugs and antiangiogenic agents such as different monoclonal antibodies. It is for this reason that the term bisphosphonates related osteonecrosis of the jaws (BRONJ) has been replaced by medication related osteonecrosis of the jaws (MRONJ) [1, 2].

This entity is characterized by the presence of necrotic bone that does not heal in a 8-week period in patients who have received treatment with these drugs without having history of radiotherapy in the neck/head region [1]. ONJ is believed to be produced by the suppression of bone remodelling with consequent ischemia and bone sclerosis. The prevalence is higher in patients who are treated intravenously $(0-0.348 \%)$, although the importance of oral administration should not be underestimated $(0.001-0.10 \%)[2,3]$.

The risk factors are locals, as performing dentoalveolar surgeries such as tooth extractions, infections or the use of removable prostheses [4,5]; and systemic, as route of administration, cumulative dose, duration of the treatment, disease for which the drug was prescribed, concomitant therapies such as chemotherapy and systemic conditions such as diabetes and rheumatoid arthritis [5,6]. Nonetheless, necrotic bone exposure can also occur spontaneously. MRONJ is more prevalent in the posterior area of the mandible, due to the anatomical characteristics of this bone, with lower vascularization compared to the maxillary bone [6].

MRONJ diagnosis is basically clinical, although bone sclerosis could be seen in the affected areas in a routine radiographic examination. However, conventional radiographic diagnosis is not currently considered to be the best technique for their detection, being Computed tomography $(\mathrm{CT})$ essential [5, 7]. It is important to make a differential diagnosis with other pathologies such as osteomyelitis, osteorradionecrosis, oral squamous cell carcinoma or osteosarcoma [3]. Table 1 shows the most commonly used classification [5] proposed by the American Association of Oral and Maxillofacial Surgeons (AAOMS) for MRONJ and treatment recommendations according the staging $[8,9]$ : 
Medication-related osteonecrosis of the jaws (MRONJ). Case report and update.

\begin{tabular}{|c|c|c|}
\hline Stage & \multicolumn{1}{|c|}{ Clinical diagnosis } & Treatment \\
\hline At risk & $\begin{array}{l}\text { No areas of bone necrosis in patients who have been } \\
\text { treated with oral or intravenous BF. }\end{array}$ & No treatment. Patient's education. \\
\hline Stage 0 & $\begin{array}{l}\text { No clinical evidence but with non-specific clinical } \\
\text { and radiological symptoms. }\end{array}$ & Expectant. \\
\hline Stage 1 & $\begin{array}{l}\text { Bone exposure with necrotic bone in asymptomatic } \\
\text { patients without evidence of infection. }\end{array}$ & $\begin{array}{l}\text { Eliminate local irritants, oral hygiene measures, } \\
\text { chlorhexidine mouthrinse 0.12\% and analgesics in } \\
\text { case of pain. }\end{array}$ \\
\hline Stage 2 & $\begin{array}{l}\text { Bone exposure with necrotic bone along with } \\
\text { infection, evident pain and erythema around the exposed } \\
\text { bone area with/without purulent drainage. }\end{array}$ & $\begin{array}{l}\text { Chlorhexidine mouthrinse 0.12\%, analgesics and } \\
\text { antibiotics (minimum duration of 15 days). } \\
\text { Microbiological cultures would be helpful in } \\
\text { choosing the specific antibiotic. }\end{array}$ \\
\hline Stage 3 & $\begin{array}{l}\text { Bone exposure with necrotic bone in patients with } \\
\text { pain, infection and one or more of these signs: } \\
\text { pathological fracture, extraoral fistula, } \\
\text { oroantral/oronasal communication or osteolysis } \\
\text { extending to the mandibular lower border/maxillary } \\
\text { sinus. }\end{array}$ & $\begin{array}{l}\text { Chlorhexidine, analgesics, antibiotics and } \\
\text { surgical treatment: curettage, sequestrectomy and } \\
\text { block resections. The latter should be done with great } \\
\text { care by compromised vascularization of the bone, } \\
\text { removing the least amount of bone and manipulating } \\
\text { as little as possible the soft tissues. }\end{array}$ \\
\hline
\end{tabular}

\section{Case Report}

An 82-year-old man was referred to the Oral Medicine Department in the School of Dentistry at Complutense University of Madrid for presenting necrotic asymptomatic lesions in the maxilla. The patient was being treated for bone metastasis due to a prostate cancer diagnosed 11 years ago with Zometa ${ }^{\circledR}$ monthly (Zoledronic acid), Zoladex ${ }^{\circledR}$ quarterly (Goselerina acetate) and Prednisone. In addition, due to the presence of other pathologies, the patient is being treated with natecal, escitalopram, pantoprazole, acetylsalicylic acid and enalapril.

The patient had a poorly adapted upper removable partial denture. Once it was removed, multiple ulcerous lesions with necrotic exposed bone could be seen. In the middle third of the palate the patient presented a lesion of approximately $2 \mathrm{~cm}$ in diameter (Fig. 1.a). In the right upper alveolar ridge at premolar-molar level, a similar lesion was present (Fig. 1.b). In the upper left alveolar ridge, two lesions of approximately $1.5 \mathrm{~cm} \mathrm{x}$ $0.5 \mathrm{~cm}$ and $1 \mathrm{~cm} \times 0.5 \mathrm{~cm}$ respectively were present (Fig. 1.c). All these lesions presented high levels of plaque and inflammation of the adjacent mucosa.

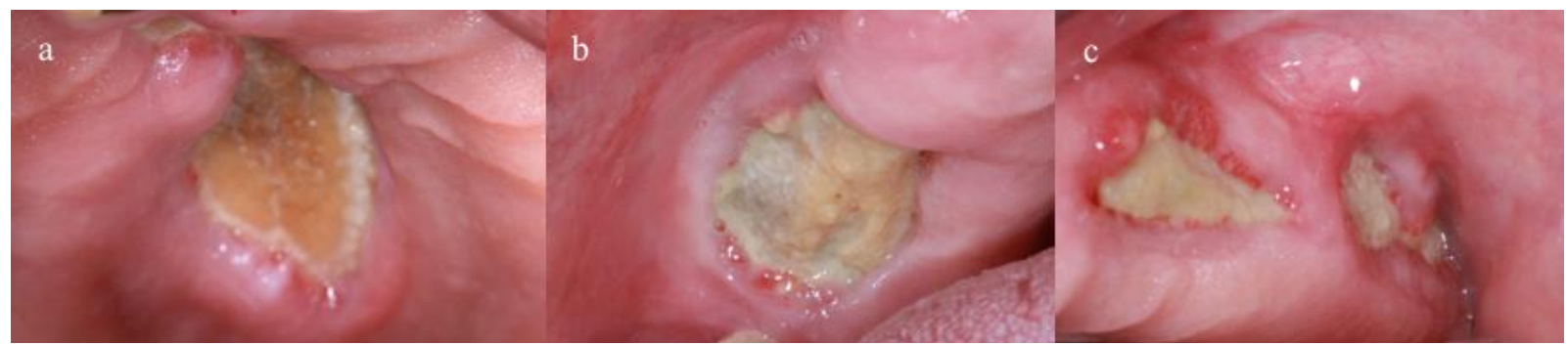

Figure 1. Clinical image of the intraoral lesions.

In the mandible, large infragingival caries was present at 46 (Fig. 2). The tooth also presented mobility, for which an extraction would be indicated.

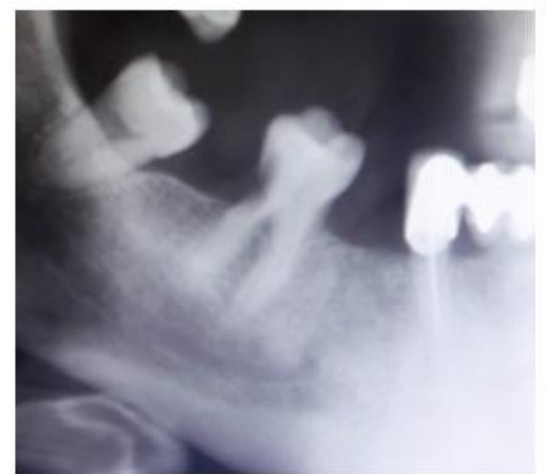

Figure 2. Radiographic visualization of tooth decay 46. 
Orthopantomography and Cone beam computered tomography. (CBCT) (Fig. 3a, 3b) showed radiopaque images in the maxillary bone compatible with osteolysis.

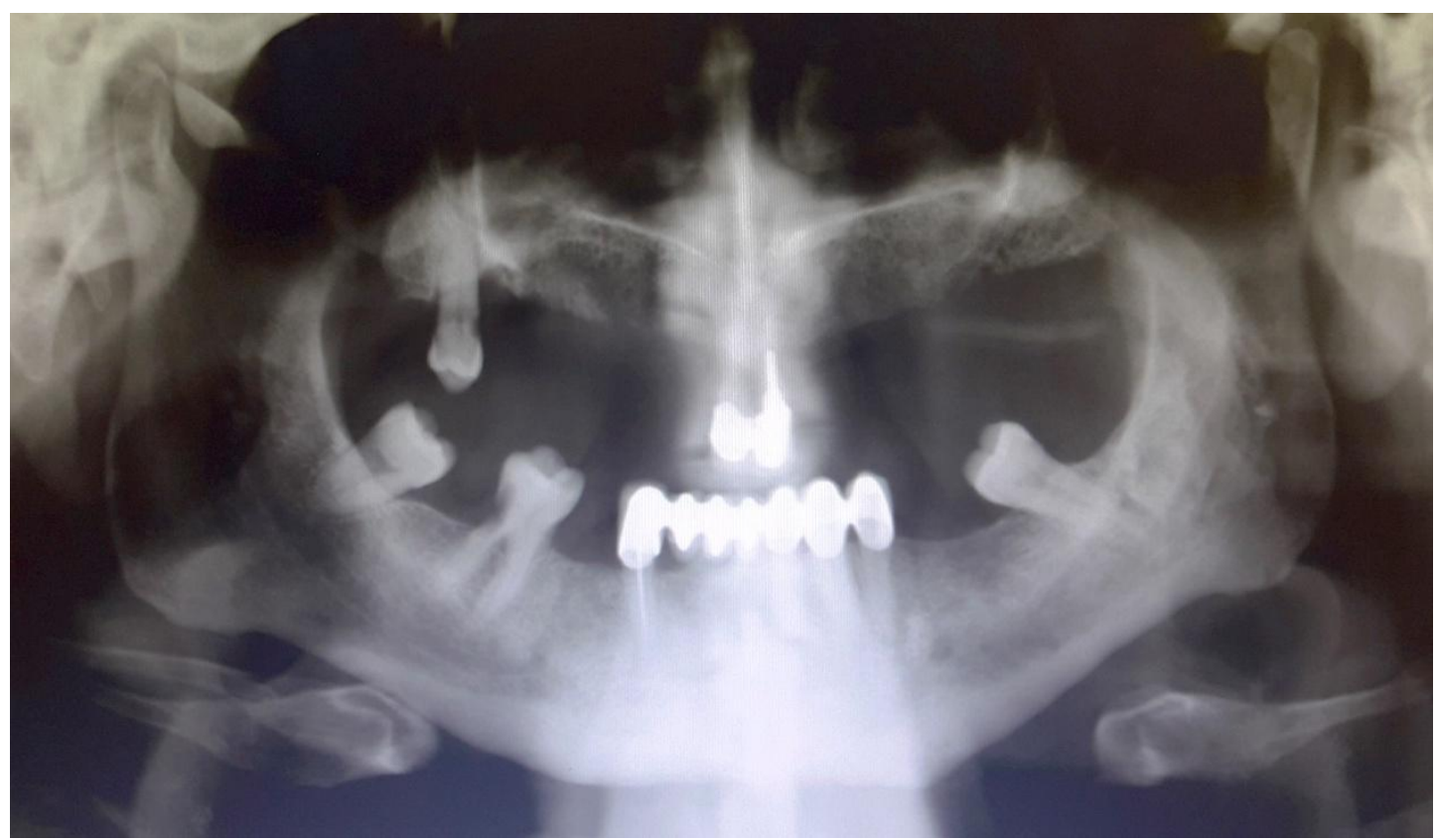

Figure 3a. Panoramic radiograph made on the first visit.
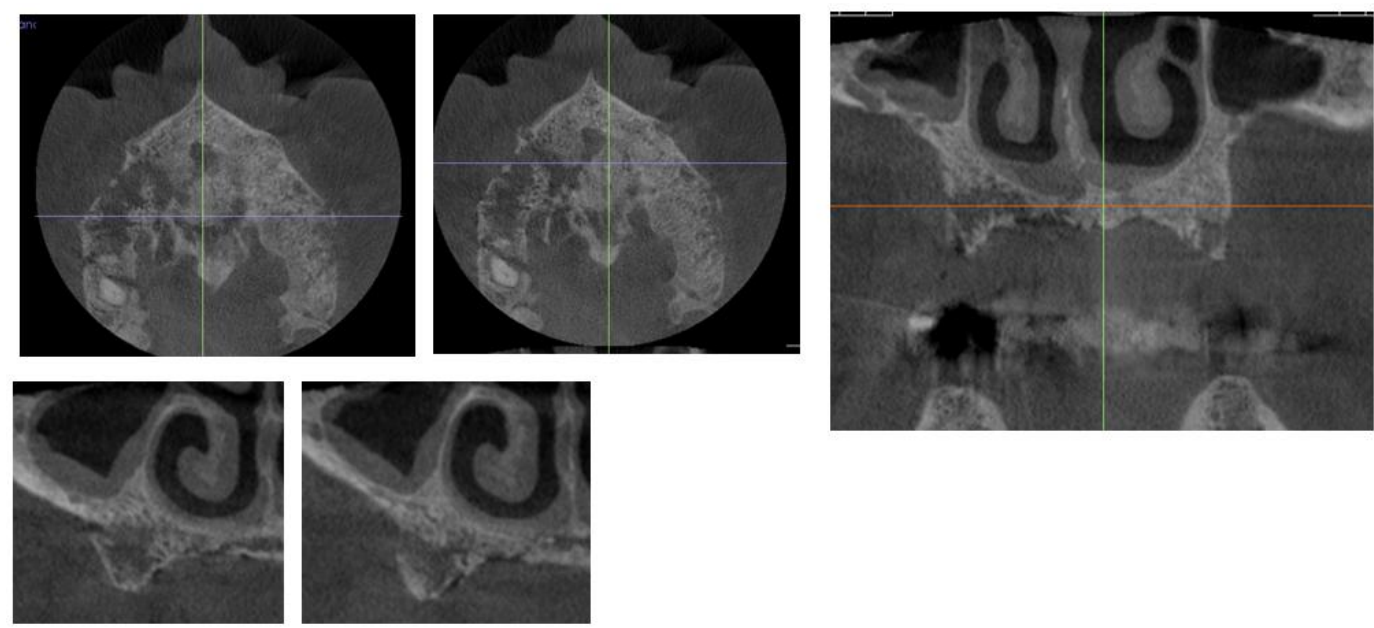

Figure 3b. Cone beam computered tomography. (CBCT)

A diagnosis of BRONJ was made due to the use of a removable prosthesis and the intake of Prednisone and Zoladex, drugs that can delay the healing of the tissues.

As it was a stage 1 (presence of exposed bone without symptoms nor signs of infection), it was decided to follow the recommendations of the AAOMS and the SECOM. A conservative treatment was carried out with rigorous dental hygiene to maintain plaque free areas of the lesion, chlorhexidine rinses at $012 \% 2$ times/day to prevent infection and topical application of chlorhexidine gel at $012 \% 2$ times/day to achieve a more local effect. In addition, it was advised not to use the superior prosthesis and, a root canal was performed on the tooth 46 since it is being treated with BFs and no surgery can be performed. An oncologic consultant was made to explain the oral lesions and an analysis was requested to see if the tumour markers were within a suitable range. Considering that cancer and bone metastases were controlled, it was decided to withdraw treatment with Zometa ${ }^{\circledR}$. Reviews were scheduled every 15 days to carry out an adequate control of the pathology and the different therapeutic alternatives available for the case were studied (laser therapy, hyperbaric oxygen administration, the application of L-PRF membranes). Finally, it was decided, because of the clinical evidence in the control of this pathology, to submit the patient to a treatment with hyperbaric oxygen. The patient was referred to Central Hospital of Defense "Gómez Ulla" (Madrid) and is currently in the waiting list for oxygen therapy. 
In one of the reviews, it was possible to see how the patient had an intraoral fistula with suppuration (Fig. 4), and it was decided to start an antibiotic therapy with amoxicillin and clavulanic acid $2 \mathrm{~g} / 125 \mathrm{mg}$ for 15 days, as recommended by the SECOM [10].

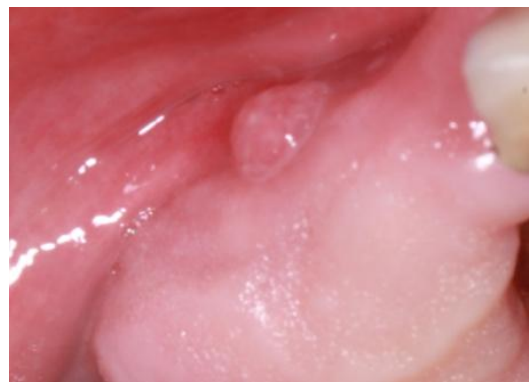

Figure 4. Intraoral fistula present in the vestibule of the first quadrant.

After 10 days a new review was performed and it was observed how the infection was controlled. However, a cavity in the bone in the necrotic lesion of the first quadrant (Fig. 5) covered by food remained. It was cleaned using physiological saline loaded in a syringe and all the residues were removed. Finally, once it was completely cleaned, the sinus membrane was seen through the cavity, and orosinusal communication was diagnosed (stage 3).

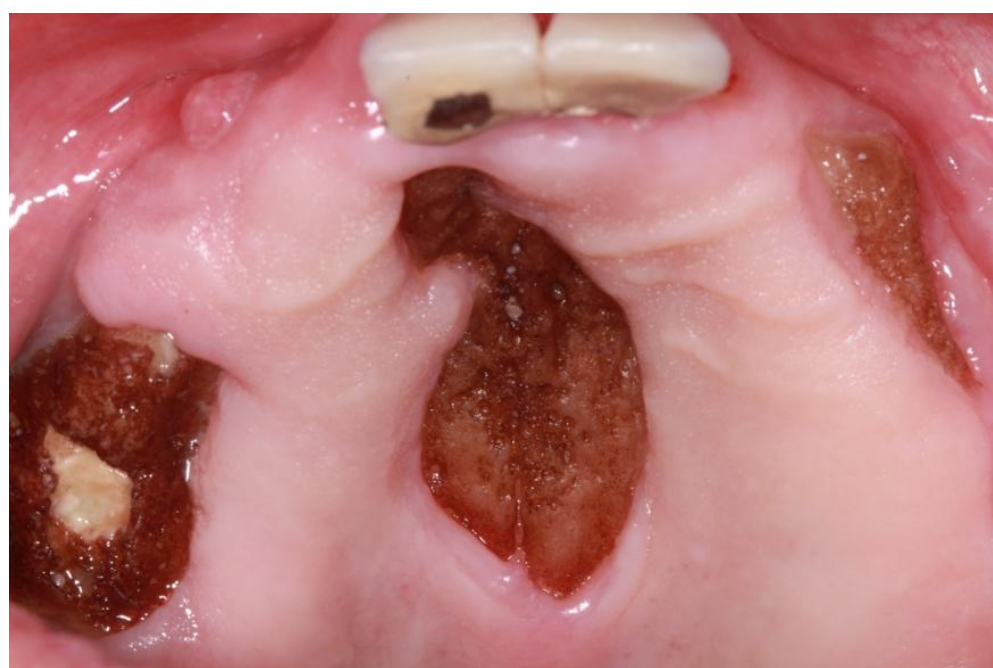

Figure 5. Image showing the cavity in the necrotic lesion of the first quadrant.

Finally, the patient was referred to the maxillofacial specialist, since, according to the current recommendations, in a stage 3 a resective maxillary surgery is needed in order to eliminate all necrotic tissue and leave only healthy bone surrounding. Surgical treatment has reported up to $92 \%$ success rates [9].

\section{Discussion}

Currently, there is controversy about what treatment should be performed in these patients, as there is no fully effective treatment. Different organisms such as the AAOMS or the SECOM advise that patients who do not present symptomatology should receive a conservative treatment, leaving the surgical treatment for those patients presenting more advanced lesions.

Even though surgical therapy reports higher success rates than other available therapies, the current trend is to use less invasive techniques that have been shown effective in a large number of cases. Among these alternatives, we find low-power laser, hyperbaric oxygen, ozone therapy, stem cell immunotherapy, teriparatide administration or the use of autologous leukocyte and platelet rich fibrin (L-PRF) or other kind of platelet concentrates like pure platelet rich plasma P-PRP, known as plasma rich in grow factors PGRF.

Low-power laser modulates cell metabolism, improves wound healing, and decreases pain. Because of this, different studies have shown effective results in the treatment of ONJ, especially when used in combination with drugs and minimally invasive surgery $[11,12]$. 
Oxygen administered in hyperbaric chambers at a concentration of $100 \%$, accelerates the differentiation of osteoblasts, stimulates angiogenesis and exerts restorative action of the bone tissue. Different authors have confirmed that this therapy prevents bone loss in BRONJ, so it seems to be a good treatment when applied during the early stages $[13,14]$. Ozone has been showed to repair necrotic bone lesions. For this reason, its topical administration seems to be beneficial, which may allow patients to lead a normal life [15].

Stem cells have the capacity for self-renewal and differentiation, so it is very useful in repairing damaged tissues. However, their use in the ONM have only been performed in animals, obtaining promising results. Nonetheless, more studies are needed to demonstrate its true efficacy $[16,17]$. Teriparatide is an analogue of parathyroid hormone approved in the treatment of osteoporosis for its ability to stimulate bone formation. It acts by stimulating osteoblasts, which significantly increases bone volume and density. In addition, it has the ability to increase the number of blood vessels within the bone marrow and at sites close to bone formation. Several authors have proposed this hormone as treatment, most of them with very satisfactory results $[18,19,20]$.

L-PRF is a leukocyte and platelet concentrate meshed within a slowly polymerized fibrin network, the second generation of platelet concentrates, capable of releasing growth factors and proteins involved in wound healing by promoting cell proliferation and differentiation, stimulating bone remodelling and soft tissue healing. Several studies concluded that the use of L-PRF seemed to be promising, especially when morphogenetic protein-2 (BMP-2) is also applied [21,22,23].

\section{Conclusion}

At present, the treatment of MRONJ should be done depending on the stage of the disease, being preferable to start with a conservative treatment. New promising and less invasive treatment alternatives are emerging, improving the patient's quality of life. Further research is needed to verify these treatment modalities as alternatives to the surgical approach for advanced stages of the disease.

There are no acknowledgements.

\section{Acknowledgements}

\section{References}

[1] Pichardo SE, Richard van Merkesteyn JP. Bisphosphonate-related osteonecrosis of the jaws: spontaneous or dental origin? Oral Surg Oral Med Oral Pathol Oral Radiol. 2013; 116: 287-292.

[2] Kumar V, Shahi AK. Nitrogen containing bisphosphonates associated osteonecrosis of the jaws: A review for past 10 year literature. Dent Res J. 2014; 11: 147-153.

[3] Khan AA, Morrison A, Hanley DA, Felsenberg D, McCauley LK, O'Ryan F, et al. Diagnosis and management of osteonecrosis of the jaw: a systematic review and international consensus. J Bone Miner Res. 2015; 30: 3-23.

[4] Campisi G, Fedele S, Fusco V, Pizzo G, Di Fede O, Bedogni A. Epidemiology, clinical manifestations, risk reduction and treatment strategies of jaw osteonecrosis in cancer patients exposed to antiresorptive agents. Future Oncol. 2014; 10: $257-275$.

[5] Ruggiero SL, Dodson TB, Fantasia J, Goodday R, Aghaloo T, Mehrotra B, et al. American Association of Oral and Maxillofacial Surgeons position paper on medication-related osteonecrosis of the jaw - 2014 update. J Oral Maxillofac Surg. 2014; 72: 19381956.

[6] Thumbigere-Math V, Tu L, Huckabay S, Dudek AZ, Lunos S, Basi DL, et al. A retrospective study evaluating frequency and risk factors of osteonecrosis of the jaw in 576 cancer patients receiving intravenous bisphosphonates. Am J Clin Oncol. 2012; 35: 386392.

[7] Margaix-Muñoz M, Bagán J, Poveda-Roda R. Intravenous bisphosphonate-related osteonecrosis of the jaws: influence of coadjuvant antineoplastic treatment and study of buccodental condition. Med Oral Patol Oral Cir Bucal. 2013; 18: e194-e200.

[8] Yoneda T, Hagino H, Toshitsugu Sugimoto T, Ohta H, Takahashi S, Soen S, et al Antiresorptive agent-related osteonecrosis of the jaw: Position Paper 2017 of the Japanese Allied Committee on Osteonecrosis of the Jaw. J Bone Miner Metab. 2017. doi: 10.1007/s00774-016-0810-7. [Epub ahead of print]

[9] Rodríguez-Lozano FJ, Oñate-Sánchez RE. Treatment of osteonecrosis of the jaw related to bisphosphonates and other antiresorptive agents. Med Oral Patol Oral Cir Bucal. 2016; 21(5): 595-600.

[10] Junquera LM, Acero J. Diagnosis, prevention, and treatment of bisphosphonate-associated osteonecrosis of the jaw. Recommendations of the Spanish Society of Oral and Maxillofacial Surgery (SECOM). Rev Esp Cirug Oral y Maxilofac. 2008; 30(3): 145-156.

[11] Blessmann Weber JB, Stifelman Camilotti R, Estér Ponte M. Efficacy of laser therapy in the management of bisphosphonaterelated osteonecrosis of the jaw (BRONJ): a systematic review. Lasers Med Sci. 2016; 31: 1261-1272.

[12] Latifyan S, Genot MT, Klastersky J. Bisphosphonate-related osteonecrosis of the jaw: a review of the potential efficacy of low-level laser therapy. Support Care Cancer. 2016; 24: 3687-3693.

[13] Ceponis P, Keilman C, Guerry C, Freiberger JJ. Hyperbaric oxygen therapy and osteonecrosis. Oral Dis. 2016; doi: 10.1111/odi.12489.

[14] Al Hadi H, Smerdon GR, Fox SW. Hyperbaric oxygen therapy accelerates osteoblast differentiation and promotes bone formation. J Dent. 2015; 43: 382-388.

[15] Ripamonti CI, Cislaghi E, Mariani L, Maniezzo M. Efficacy and safety of medical ozone (O3) delivered in oil suspension applications for the treatment of osteonecrosis of the jaw in patients with bone metastases treated with bisphosphonates: Preliminary results of a phase I-II study. Oral Oncol. 2011; 47: 185-190.

[16] Matsuura Y, Atsuta I, Ayukawa Y, Yamaza T, Kondo R, Takahashi A, et al. Therapeutic interactions between mesenchymal stem cells for healing medication-related osteonecrosis of the jaw. Stem Cell Res Ther. 2016; 7: 119. 
[17] De Oliveira CC, Brizeno LAC, de Sousa FB, Mota MRL, Alves APNN. Osteonecrosis of the jaw induced by receptor activator of nuclear factor-kappa B ligand (Denosumab) - Review. Med Oral Patol Oral Cir Bucal. 2016; 21: e431-e439

[18] Lee JJ, Cheng SJ, Jeng Jh, Chiang CP, Lau hP, Kok Sh. Successful treatment of advanced bisphosphonate-related osteonecrosis of the mandible with adjunctive teriparatide therapy. Head Neck. 2011; 33: 1366-1371.

[19] Neuprez A, Rompen E, Crielaard JM, Reginster JY. Teriparatide therapy for denosumab-induced osteonecrosis of the jaw in a male osteoporotic patient. Calcif Tissue Int. 2014; 95: 94-96.

[20] Keskinruzgar A, Bozdag Z, Aras MH, Demir T, Yolcu U, Cetiner S. Histopathological effects of teriparatide in medication-related osteonecrosis of the jaw: an animal study. J Oral Maxillofac Surg. 2016; 74: 68-78.

[21] Kim JW, Kim SJ, Kim MR. Leucocyte-rich and platelet-rich fibrin for the treatment of bisphosphonate-related osteonecrosis of the jaw: A prospective feasibility study. Br J Oral Maxillofac Surg. 2014; 52: 854-859.

[22] Zhao QM, Ding YJ, Si T. Platelet-rich fibrin in plastic surgery. OA Evidence-Based Medicine. 2013; 1: 3.

[23] Park JH, Kim JW, Kim SJ. Does the Addition of Bone Morphogenetic Protein 2 to Platelet-Rich Fibrin Improve Healing After Treatment for Medication-Related Osteonecrosis of the Jaw? J Oral Maxillofac Surg. 2016. pii: S0278-2391(16)31215-0. 